\title{
Pesca exploratoria con espineles en aguas profundas en torno a la isla Robinson Crusoe (Archipiélago de Juan Fernández), Chile*
}

\author{
Patricio M. Arana y Rodrigo Vega \\ Escuela de Ciencias del Mar, Universidad Católica de Valparaíso \\ Casilla 1020, Valparaíso, Chile \\ E-mail: parana@ucv.cl
}

Recibido: 18 enero 1999; versión corregida: 4 junio 1999; aceptado: 20 octubre 1999

\begin{abstract}
RESUMEN La pesca exploratoria con espineles se efectuó en torno a la isla Robinson Crusoe del archipiélago de Juan Fernández, entre noviembre de 1996 y marzo de 1997. Esta experiencia fue desarrollada mediante el calado de espineles horizontales y verticales en estaciones posicionadas sobre 4 transectas orientadas radialmente a las islas, entre 50 y $1000 \mathrm{~m}$ de profundidad. En dicha experiencia se trabajó principalmente con anzuelos desbalanceados números $5,6,7$ y 9 .

Los principales recursos capturados en estas actividades correspondieron a: anguila de profundidad (Bassanago nielseni), breca (Cheilodactylus gayi), cabrilla de Juan Fernández (Scorpaena fernandeziana), chancharro (Helicolenus lengerichi), corvina de Juan Fernández (Umbrina reedi), jurel de Juan Fernández (Pseudocaranx chilensis), morena (Gymnotorax porphyreus), ribaldo (Mora moro), tollo de Juan Fernández (Squalus mitsukurii), pez mariposa (Pterygotrigla picta) y alfonsino (Beryx splendens). Si bien las capturas obtenidas con estos aparejos no fueron especialmente abundantes, permitieron determinar la fauna íctica de aguas profundas en torno a estas islas. Las especies que presentaron los rendimientos más altos fueron la breca y tollo de Juan Fernández, entre 50 y 200 m, y el ribaldo, entre 400 y 600 m. Entre 600 y $1000 \mathrm{~m}$ no se registró captura de peces.
\end{abstract}

Palabras claves: pesca exploratoria, espineles, archipiélago de Juan Fernández, Chile.

\section{Longline exploratory fishing survey in deep waters around Robinson Crusoe island (Juan Fernandez archipelago), Chile}

\begin{abstract}
A longline exploratory fishing around Robinson Crusoe island, belonging to the Juan Fernandez Archipelago, was conducted between November 1996 and March 1997. The experience was developed by setting horizontal and vertical longlines at stations over four radial transects, between 50 and $1000 \mathrm{~m}$ depth, using unbalanced hooks numbers 5, 6, 7 and 9 .

Main resources caught during these activities were: deep water eel (Bassanago nielseni), breca (Cheilodactylus gayi), Juan Fernandez rockfish (Scorpaena fernandeziana), croaker (Helicolenus lengerichi), Juan Fernandez corvina (Umbrina reedi), Juan Fernandez mackerel (Pseudocaranx chilensis), moray (Gymnotorax porphyreus), codling (Mora moro), Juan Fernandez dogfish (Squalus mitsukurii), robin fish (Pterygotrigla picta) and alfonsino (Beryx splendens). Even though the catch with these gears was not conspicuously abundant, it was possible to identify the deep water fauna around these islands. The species with higher yields were the breca and Juan Fernandez dogfish, between 50 and 200 $\mathrm{m}$, and codling, between 400 and $600 \mathrm{~m}$. Between 600 and $1000 \mathrm{~m}$ no fishes were recorded.
\end{abstract}

Key words: exploratory fishing, longlines, Juan Fernandez archipelago, Chile.

* Artículo generado como parte del "Programa de pesca exploratoria y experimental de recursos pesqueros alternativos a la langosta en las islas Robinson Crusoe y Santa Clara" (Proyecto FIP 95/25), financiado por el Fondo de Investigación Pesquera y realizado por la Universidad Católica de Valparaíso. 
Las islas Robinson Crusoe y Santa Clara del archipiélago de Juan Fernández, ubicadas sobre el paralelo $33^{\circ} 40^{\prime}$ de latitud sur y aproximadamente 360 millas náuticas del continente sudamericano, presentan una flora y fauna bentónica rica y diversificada, siendo algunas de sus especies de distribución exclusiva o autóctona de ese archipiélago. En el sustrato marino predominan rocas de tipo volcánico, con abundantes grietas y cuevas que albergan numerosas especies. Con relación a la fauna íctica, ésta ha sido estudiada principalmente en cuanto a sus aspectos taxonómicos. Según Sepúlveda y Pequeño (1985), se han mencionado para esta región 146 especies y seis subespecies pertenecientes a 67 familias.

En la actualidad, la actividad pesquera que se realiza en el archipiélago está centrada casi exclusivamente en la explotación de la langosta (Jasus frontalis $\mathrm{H}$. Milne-Edwards, 1837), recurso que es capturado por medio de trampas construidas artesanalmente con maderas locales. En general, la mayoría de los peces que se capturan en el archipiélago se utilizan como carnada de las trampas, destinándose únicamente una parte minoritaria de las capturas a consumo local y sólo esporádicamente se dirige la extracción a proveer a compradores ocasionales que comercializan esta pesca en el continente. Estos recursos se capturan hasta ahora con líneas de mano, espineles verticales y trampas para anguilas (Arana y Ziller, 1985).

Existen antecedentes acerca de la presencia de especies pelágicas migratorias que visitan el archipiélago en algunas estaciones del año y de recursos bentodemersales que pueden tener cierta importancia pesquera potencial. Cabe destacar, además, que la zona comúnmente explotada en el archipiélago alcanza hasta $\operatorname{los} 200 \mathrm{~m}$ de profundidad, utilizándose artes de pesca selectivos que impiden un adecuado conocimiento de otros organismos que puedan existir, incluso en dicho rango.

Tradicionalmente ha sido el bacalao de Juan Fernández (Polyprion oxygeneios Schneider, 1801) la especie íctica de mayor interés para los pescadores de estas islas (Arana y Ziller, 1985; Pavez y Oyarzún, 1985; Pizarro y Yañez, 1985), aunque se desconoce la profundidad máxima a la que se le puede encontrar en torno a estas islas. Por otra parte, en faenas de pesca exploratoria con espineles de profundidad, realizadas en el monte submarino O'Higgins ( $\left.32^{\circ} 59^{\prime} \mathrm{S}-73^{\circ} 57^{\prime} \mathrm{W}\right)$, se capturaron ribaldos (Mora moro Risso, 1810) a $400 \mathrm{~m}$ de pro- fundidad (Moreno, 1974; Cerda, 1977), desconociéndose aún si esta especie se encuentra en las islas Robinson Crusoe y Santa Clara. Dadas las problemáticas antes indicadas y a que todavía se desconoce qué recursos pueden estar presentes en aguas profundas en torno a estas islas, se ha considerado de alto interés el conocer la fauna íctica entre los 200 y $1000 \mathrm{~m}$ de profundidad.

Junto con lo anterior, es también importante el considerar que los desembarques anuales de langosta se han mantenido por varios años en los niveles más bajos registrados en la historia de esta pesquería, lo que constituye un hecho de permanente preocupación, ya que ello enfrenta a un futuro incierto respecto al desarrollo social y económico de los habitantes de esta posesión insular. De allí el interés por identificar alternativas de explotación que permitan diversificar la extracción de recursos. Al respecto, el presente trabajo tuvo como objetivo establecer la existencia de recursos ícticos factibles de explotar, ya sea como especies principales o en forma complementaria a la extracción de langosta.

Las labores de pesca exploratoria se realizaron alrededor de isla Robinson Crusoe, considerando estaciones de trabajo posicionadas sobre cuatro transectas trazadas perpendicularmente a esta isla, tratando de cubrir de esta forma distintos sectores de la plataforma y talud en torno a esta isla. Estas se ubicaron frente a Puerto Francés (transecta 1), islote Juanango (transecta 2), Bahía El Padre (transecta 3) e islote El Verdugo (transecta 4). En cada una de ellas, se establecieron 9 estaciones ubicadas respectivamente sobre los veriles de 50, 100, 200, 300, 400, 500, 600, 800 y $1000 \mathrm{~m}$ de profundidad (Fig. 1).

Debido a la dificultad encontrada en la localidad para operar con una sola embarcación, se trabajó con las chalupas de alta mar "Ariki-Ruku" (s/n), "Clara Margarita" ( $\left.\mathrm{N}^{\circ} 11\right)$, "Independencia" $\left(\mathrm{N}^{\circ} 14\right)$, "Miriam" (N²1), "Libertad" (N²9), "Guaiteca" (N54), "Norma" ( $\left.{ }^{\circ} 55\right)$, "Matilde" ( $\left.\mathrm{N}^{\circ} 65\right)$ y "Kibalión" ( $\left.\mathrm{N}^{\circ} 2310\right)$, así como también con la lancha "Natalia" $\left(\mathrm{N}^{\circ} 63\right)$. Todas ellas corresponden a embarcaciones artesanales, de 8 a $10 \mathrm{~m}$ de eslora total.

Para realizar las experiencias de pesca con espineles horizontales y verticales, las embarcaciones antes indicadas fueron equipadas con un virador hidráulico de doble plato, dispuesto en posición horizontal, accionado por un motor bencinero de 6,5 HP. Además, las chalupas se equiparon con 
posicionador satelital (GPS) y ecosonda con alcance superior a $1000 \mathrm{~m}$. En cada salida junto a la tripulación habitual de dos o tres pescadores, se embarcó un profesional encargado de operar los instrumentos electrónicos, recolectar la información biológico-pesquera y supervisar las labores efectuadas en terreno. En algunas oportunidades se agregó otro técnico o profesional para colaborar con las tareas de abordo.

En las experiencias se utilizaron dos tipos de espineles: verticales y horizontales. En ambos casos se utilizó un solo orinque; en su extremo se dispone la línea madre adicionada, donde van montados los reinales con su respectivo anzuelo. Los espineles verticales reciben ese nombre en atención a que la línea madre queda en forma vertical sobre el fondo, mientras que en el caso de los espineles horizontales la línea madre queda calada en forma paralela al fondo marino.
En la construcción de los aparejos se utilizaron dos tipos de cabos: en el orinque PE torcido de 5 $\mathrm{mm}$ de diámetro y construcción $\mathrm{Z}$ y en la línea madre PE torcido de $3 \mathrm{~mm}$ de diámetro y construcción S. En los reinales se empleó PA monofilamento de 0,6 $\mathrm{m}$ de largo y $1 \mathrm{~mm}$ de diámetro, separados entre sí por $1,83 \mathrm{~m}$ ( 1 braza).

En los espineles verticales se utilizó un número total de anzuelos que fluctuó entre 20 y 40 unidades por lance. Los anzuelos utilizados correspondieron a modelos desbalanceados, de marca Mustad Kirby y Maruto, números 5, 6, 7 y 9, dispuestos en forma aleatoria a lo largo de la línea (Fig. 2A). Cabe señalar que inicialmente se utilizaron anzuelos números 2 y 3 , a fin de determinar su desempeño ante la posibilidad de capturar peces de gran tamaño; sin embargo, éstos fueron desechados al comprobar que únicamente se obtenían ejemplares de tallas medianas y pequeñas.

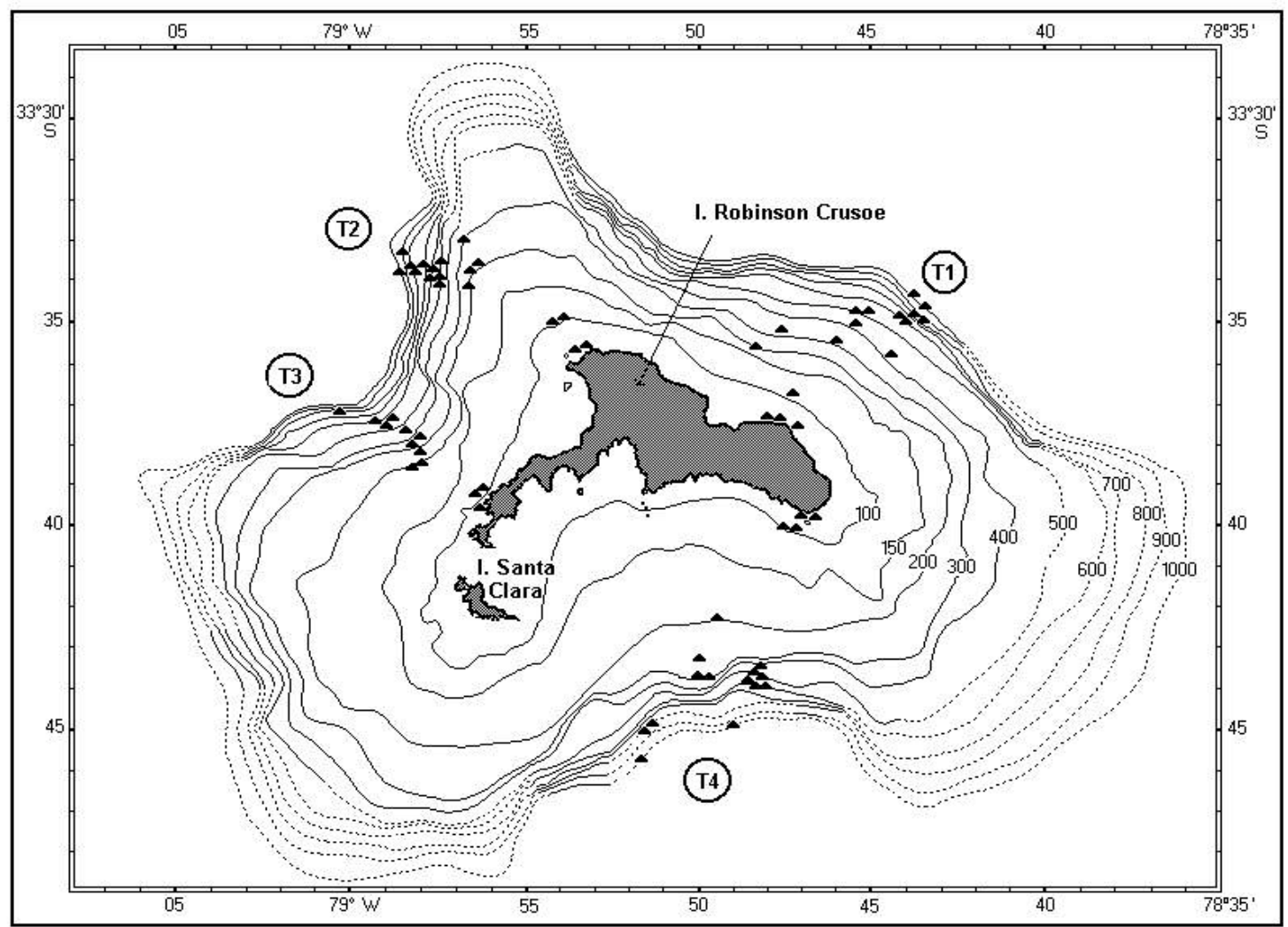

Figura 1. Transectas (T) y estaciones ( $z$ ) donde fueron calados espineles durante la pesca exploratoria. Figure 1. Transects $(\mathrm{T})$ and stations $(\tau)$ where longlines were set during the exploratory fishing. T1: Puerto Francés, T2: Islote Juanango, T3: Bahía El Padre, y T4: Islote El Verdugo. 
En la construcción de los espineles horizontales se utilizaron los mismos materiales empleados en los espineles verticales (Fig. 2B). Los anzuelos utilizados correspondieron igualmente a Mustad Kirby y Maruto, desbalanceados, de los números 5, 6, 7 y 9 , en cantidad que variaron entre 20 y 120 unidades por espinel.

En ambos tipos de espineles el sistema de flotación fue adecuado a las condiciones imperantes en la zona de calado (estado del mar y corrientes). Con este fin, se utilizaron boyas elipsoides y rígidas de $30 \mathrm{~cm}$ de diámetro como elementos boyantes de mediagua y flotadores Vinycon de $5 \mathrm{~kg}$ de boyantez (2 a 4 unidades) en superficie. Como elemento señalizador se usaron banderolas de 1,6 $\mathrm{m}$ de largo provistas de 2 flotadores Vinycon y una cadena en su base, como lastre. Así también, para eliminar la posibilidad de deriva del aparejo, el fondeo se efectuó con piedras envueltas en paños de red.

El régimen operativo empleado en las faenas de pesca puede ser descrito de la siguiente manera: zarpe alrededor de las 08:00 hr, captura de carnada en la zona costera hasta completar la cantidad requerida como carnada de los espineles. Esta carnada estuvo conformada básicamente por jurel de Juan Fernández (Pseudocaranx chilensis), añadiéndose ocasionalmente pampanito (Scorpis chilensis Guichenot, 1848), pulpo (Octopus sp.) y chancharro

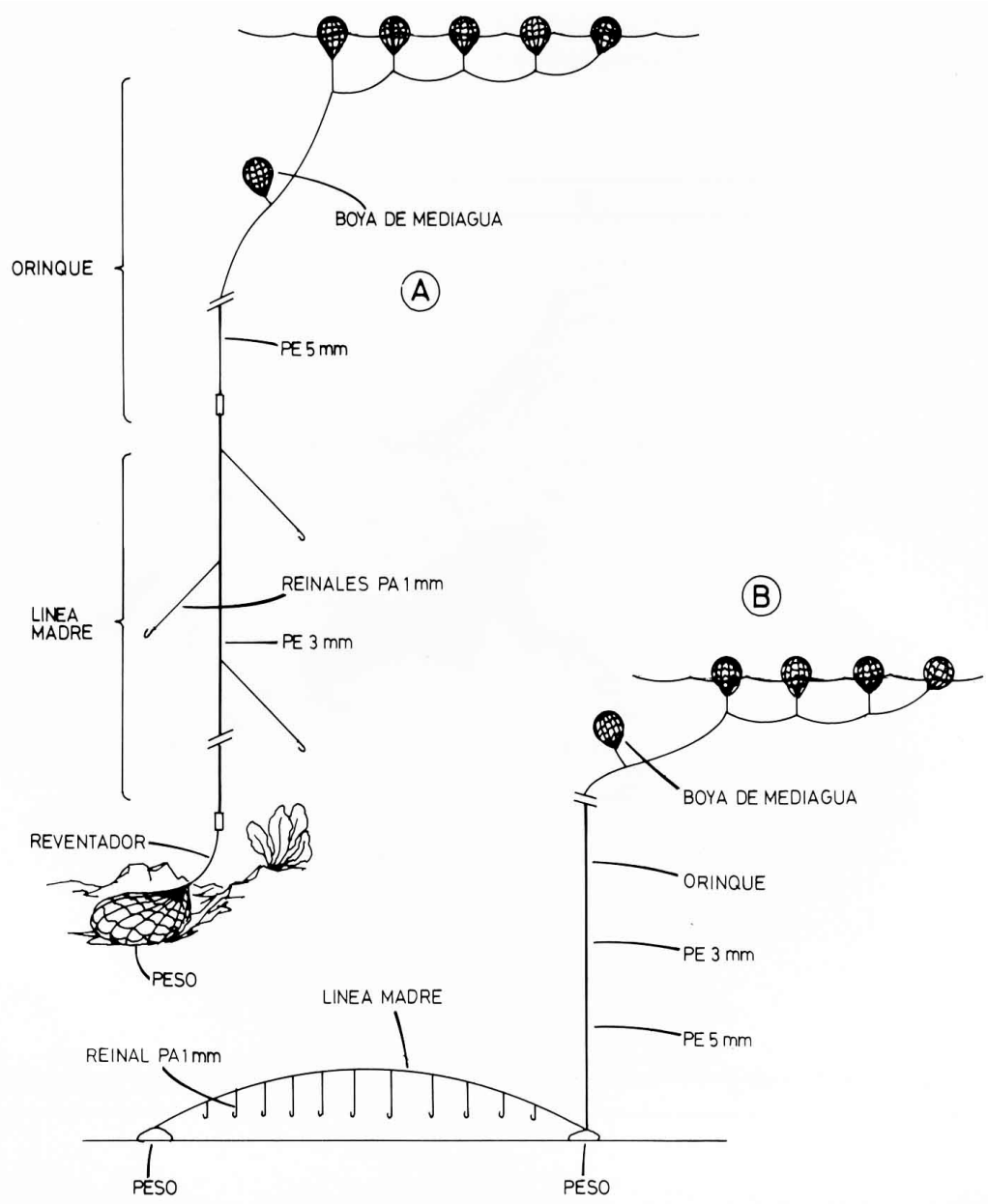

Figura 2. Espineles utilizados en la pesca exploratoria: A: Espinel vertical y B: Espinel horizontal. Figure 2. A: Vertical, and B: Horizontal longline, used during the exploratory fishing. 
(Helicolenus lengerichi Norman, 1937). Seguidamente, se procedió a calar los espineles de acuerdo al programa de muestreo, calando y virando los aparejos en forma sucesiva en cada estación. Esta rutina se extendía hasta aproximadamente las 18:00 hr.

En cada lance se llevó registro de fecha, posición geográfica, profundidad de trabajo, tipo de carnada utilizada, aparejo de pesca empleado y características específicas del mismo. Además, se llevó control in situ del tamaño y peso total de los ejemplares capturados. Los rendimientos se calcularon por especie, como el cociente entre el peso capturado y el número de anzuelos empleados en el respectivo lugar de pesca. Los peces fueron medidos con un ictiómetro, registrando la longitud total al centímetro inferior y el peso total fue determinado en forma individual con ayuda de un dinamómetro. Para la ubicación geográfica del lugar donde se calaron los espineles se utilizaron simultáneamente "marcas a tierra" y demarcaciones con GPS. Paralelamente, se obtuvieron muestras de los peces extraídos, los que posteriormente fueron conservados en formalina para su identificación taxonómica.

Los trabajos de pesca exploratoria con espineles verticales se realizaron entre el 11 de noviembre de 1996 y el 24 de marzo de 1997. En este período se efectuaron 36 salidas de pesca, que cubrieron 34 de las 36 estaciones programadas, acumulando 394 horas efectivas de pesca. En dichas faenas se caló un total de 998 anzuelos (Tabla 1).

El régimen operativo se adecuó al estado del mar imperante en los sitios donde se realizaron las faenas de pesca. La principal modificación correspondió a la disminución en el tiempo de reposo de los espineles, el que fue reducido progresivamente hasta un tiempo aproximado de $2 \mathrm{hr}$ por lance, especialmente en las estaciones más profundas, debido a la progresiva disminución en la efectividad de la carnada y a la posibilidad de extravío de material, ya sea por corte del orinque o por el hundimiento de los boyerines debido a la acción de las corrientes.

El cabo torcido de PE, de construcción $\mathrm{Z}$ de 5 $\mathrm{mm}$ de diámetro presentó mayor adherencia al eje del disco virador, mientras que el cabo de PE torcido de $3 \mathrm{~mm}$ de diámetro de construcción $\mathrm{S}$, no dio buenos resultados al destorcerse con facilidad y enrollarse, dificultando las operaciones a bordo de la embarcación. Junto con ello, debido a las corrientes imperantes en el lugar en los primeros meses de trabajo provocaron que los flotadores utilizados inicialmente se hundieran, comprimiéndose con la pre- sión y absorbiendo agua, por lo que disminuyeron su capacidad de flotación, no pudiendo soportar el peso del material. Por ello, se cambiaron los boyerines por boyas rígidas y se eliminó el uso de banderolas.

Con relación a los anzuelos utilizados, se comprobó que los modelos de marca Mustad Kirby presentaron mejor desempeño, debido a una menor deformación y mayor resistencia a la corrosión. También se observó en el transcurso de las pruebas que las capturas tendieron a concentrarse en el sector inferior de la línea de pesca. Por esa razón, se disminuyó la cantidad de anzuelos calados por espinel, consiguiéndose con ello menores pérdidas de material, un menor requerimiento de carnada y reducción en las posibilidades de enredo.

La captura total obtenida con este aparejo correspondió a 42 ejemplares, con un peso total de 44,9 $\mathrm{kg}$. Las capturas se obtuvieron únicamente en el rango entre 50 y $600 \mathrm{~m}$, siendo las estaciones ubicadas frente al islote Juanango (transecta 2) donde se obtuvo la mayor parte de la pesca $(60,4 \%)$. En la transecta correspondiente a Bahía El Padre (transecta 3 ) se registró el 19,5\% de las capturas en peso, mientras que en las ubicadas perpendicularmente a Puerto Francés (transecta 1) e islote El Verdugo (transecta 4) las capturas correspondieron respectivamente al $10,8 \%$ y $9,2 \%$ del peso total (Tabla 2 ).

Los peces capturados con mayor frecuencia y que aparecieron en casi todas las transectas fueron el pez mariposa (Pterygotrigla picta Günther, 1880), la breca (Cheilodactylus gayi Kner, 1865) y el ribaldo (Mora moro), con 12, 11 y 6 ejemplares, respectivamente. Otros peces que se encontraron en menor cantidad fueron el chancharro (Helicolenus lengerichi) con 5 unidades, cabrilla de Juan Fernández (Scorpaena fernandeziana Steindachner, 1875) y alfonsino (Beryx splendens Lowe, 1833) con 4 ejemplares cada uno (Tabla 2). Cabe hacer notar que ejemplares de cabrilla de Juan Fernández se pescaron exclusivamente en la transecta 1 (Puerto Francés), al mismo tiempo que en este lugar no se extrajeron brecas. Así, también, llamó la atención la ausencia de capturas de ribaldo en las estaciones ubicadas en los sectores oeste y sur de estas islas (Bahía El Padre e islote El Verdugo).

De acuerdo al tipo de recurso extraído por profundidad, se determinó que la mayor variedad se encontró entre 100 y $300 \mathrm{~m}$. En dicho rango batimétrico los peces capturados correspondieron a chancharro, breca, pez mariposa, cabrilla y 
Tabla 1. Número de anzuelos calados en espineles verticales por estación y transecta de trabajo.

Table 1. Number of hooks set in vertical longlines by station and working transect.

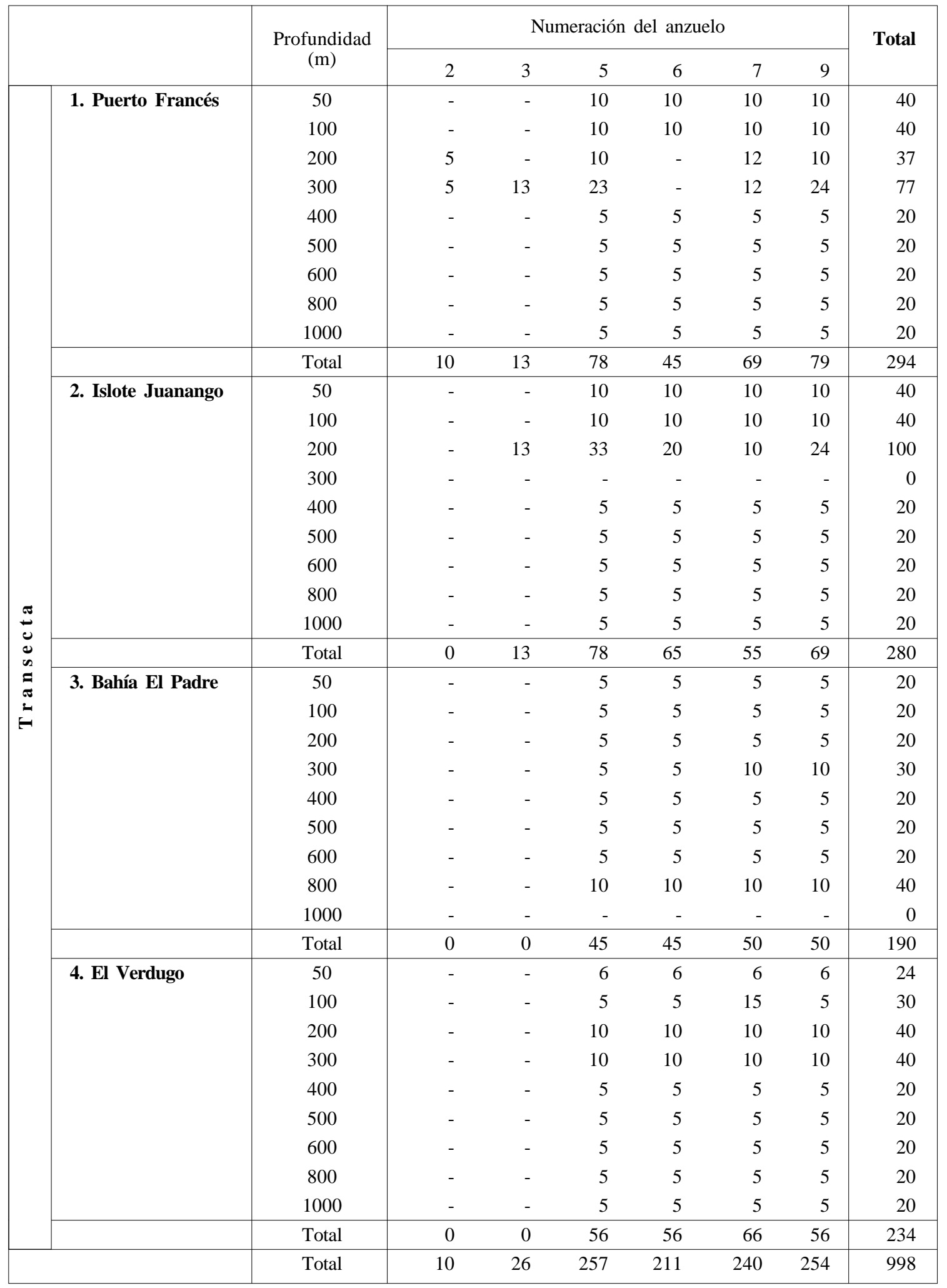


Tabla 2. Rendimiento relativo (g/anzuelo) de recursos capturados en la pesca exploratoria con espineles verticales según la numeración del anzuelo utilizado.

Table 2. Relative yield (g/hook) by resource caught during the exploratory fishing with vertical longlines, by station (depth) and number of the hook.

\begin{tabular}{|c|c|c|c|c|c|c|c|}
\hline Transecta & \multicolumn{2}{|c|}{$\begin{array}{l}\text { Profun- } \\
\text { didad } \\
\text { (m) }\end{array}$} & $\begin{array}{c}\text { Numeración } \\
\text { del anzuelo }\end{array}$ & $\begin{array}{l}\text { Cantidad } \\
\text { anzuelos }\end{array}$ & $\begin{array}{r}\text { Capt } \\
\text { Individuos }\end{array}$ & $\begin{array}{l}\text { ura } \\
\text { Peso } \\
(\mathrm{kg})\end{array}$ & $\begin{array}{c}\text { Rendimiento } \\
\text { (g/anzuelo) }\end{array}$ \\
\hline \multirow[t]{4}{*}{ 1. Puerto Francés } & \multirow[t]{3}{*}{300} & \multirow{3}{*}{$\begin{array}{l}\text { Cabrilla de } \\
\text { Juan Fernández } \\
\text { Chancharro }\end{array}$} & 7 & 12 & 2 & 0,70 & 58,3 \\
\hline & & & 9 & 24 & 2 & 1,55 & 64,6 \\
\hline & & & 9 & 24 & 2 & 1,70 & 70,8 \\
\hline & 600 & Ribaldo & 5 & 5 & 1 & 0,92 & 184,0 \\
\hline \multicolumn{3}{|r|}{ Total } & & 65 & 7 & 4,87 & \\
\hline \multirow[t]{13}{*}{ 2. Islote Juanango } & \multirow[t]{2}{*}{100} & \multirow[t]{2}{*}{ Pez mariposa } & 6 & 10 & 1 & 1,00 & 100,0 \\
\hline & & & 7 & 10 & 1 & 0,60 & 60,0 \\
\hline & \multirow[t]{6}{*}{200} & Chancharro & 5 & 33 & 1 & 0,50 & 15,2 \\
\hline & & \multirow[t]{4}{*}{ Breca } & 5 & 33 & 2 & 2,80 & 84,8 \\
\hline & & & 6 & 20 & 1 & 1,20 & 60,0 \\
\hline & & & 7 & 10 & 3 & 5,40 & 540,0 \\
\hline & & & 9 & 24 & 1 & 2,60 & 108,3 \\
\hline & & Alfonsino & 6 & 20 & 1 & 2,20 & 110,0 \\
\hline & \multirow[t]{2}{*}{500} & \multirow[t]{2}{*}{ Ribaldo } & 5 & 5 & 1 & 1,60 & 320,0 \\
\hline & & & 6 & 5 & 1 & 2,20 & 440,0 \\
\hline & \multirow[t]{3}{*}{600} & \multirow[t]{3}{*}{ Ribaldo } & 5 & 5 & 1 & 1,60 & 320,0 \\
\hline & & & 6 & 5 & 1 & 2,20 & 440,0 \\
\hline & & & 7 & 5 & 1 & 3,20 & 640,0 \\
\hline \multicolumn{4}{|c|}{ Total } & 185 & 16 & 27,10 & \\
\hline \multirow[t]{8}{*}{ 3. Bahía El Padre } & \multirow[t]{2}{*}{50} & Breca & 5 & 5 & 1 & 2,20 & 440,0 \\
\hline & & Alfonsino & 7 & 5 & 1 & 0,20 & 40,0 \\
\hline & 200 & Pez mariposa & 5 & 5 & 2 & 0,45 & 90,0 \\
\hline & \multirow[t]{4}{*}{300} & Chancharro & 7 & 10 & 1 & 0,85 & 85,0 \\
\hline & & \multirow[t]{2}{*}{ Pez mariposa } & 7 & 10 & 2 & 1,60 & 160,0 \\
\hline & & & 9 & 10 & 1 & 0,95 & 95,0 \\
\hline & & Alfonsino & 9 & 10 & 1 & 1,70 & 170,0 \\
\hline & 400 & Chancharro & 7 & 5 & 1 & 0,82 & 164,0 \\
\hline \multicolumn{4}{|c|}{ Total } & 60 & 10 & $\mathbf{8 , 7 7}$ & \\
\hline \multirow[t]{6}{*}{ 4. El Verdugo } & \multirow[t]{4}{*}{100} & Pez mariposa & 5 & 5 & 1 & 0,38 & 76,0 \\
\hline & & & 9 & 5 & 1 & 0,35 & 70,0 \\
\hline & & Breca & 7 & 15 & 3 & 2,70 & 180,0 \\
\hline & & Alfonsino & 7 & 15 & 1 & 0,20 & 13,3 \\
\hline & 200 & Pez mariposa & 5 & 10 & 2 & 0,37 & 37,0 \\
\hline & & & 7 & 10 & 1 & 0,15 & 15,0 \\
\hline & & Total & & 60 & 9 & 4,15 & \\
\hline & & Total & & 370 & 42 & 44,89 & \\
\hline
\end{tabular}


alfonsino. En 500 y 600 m sólo se capturó ribaldo (Tabla 2), mientras que en las estaciones de $800 \mathrm{y}$ $1000 \mathrm{~m}$ no se registraron capturas.

Al considerar globalmente la información recopilada en la pesca exploratoria con espineles verticales, se determinó que sobre los 200 m únicamente la breca presentó rendimientos de interés pesquero y bajo los $200 \mathrm{~m}$ sólo el ribaldo. En el primer recurso los rendimientos fluctuaron entre 60 y $540 \mathrm{~g} / \mathrm{an}$ zuelo y en el segundo entre 184 y 640 g/anzuelo (Tabla 2).

Las tallas de los ejemplares capturados en la pesca exploratoria con espineles verticales, correspondieron en el ribaldo a longitudes totales entre 40 y $68 \mathrm{~cm}$ y peso total entre 900 y $3200 \mathrm{~g}$. En la breca se obtuvieron ejemplares con longitud total comprendida entre 24 y $59 \mathrm{~cm}$ y peso total entre $500 \mathrm{y}$ $2600 \mathrm{~g}$. Los valores registrados en el pez mariposa variaron entre 20 y $47 \mathrm{~cm}$ de longitud total, mientras el rango de peso total estuvo comprendido entre 150 y $1200 \mathrm{~g}$.

La operación de pesca con espineles horizontales se efectuó entre el 11 de noviembre de 1996 y el 15 de marzo de 1997. En dicho período se realizó un total de 31 salidas de pesca, cubriéndose 30 de las 36 estaciones programadas. Con este aparejo se caló un total de 1500 anzuelos, cifra que se descompone en 460 anzuelos en la transecta ubicada frente a Puerto Francés (transecta 1), 470 frente al islote Juanango (transecta 2), 200 en Bahía El Padre (transecta 3) y 370 en islote El Verdugo (transecta 4), distribuidos en las estaciones entre 50 y $1000 \mathrm{~m}$ de profundidad (Tabla 3).

Inicialmente se utilizaron entre 60 y 120 anzuelos por espinel, cantidad que fue disminuida a 2040 unidades. Esta reducción se debió a la alta tasa de pérdida de material por trabazones en el fondo. $\mathrm{Al}$ igual que en el caso de los espineles verticales, se determinó que el cabo torcido de PE, de construcción $\mathrm{Z}$, de $5 \mathrm{~mm}$ de diámetro, presentó mayor adherencia al eje del disco virador. En cuanto el cabo de $\mathrm{PE}$ torcido, de construcción $\mathrm{S}$, de $3 \mathrm{~mm}$ de diámetro, no dio buen resultado, ya que se destorcía y enrollaba con facilidad.

Debido principalmente a la pérdida, tanto de efectividad de la carnada como de material de pesca por enredos y trabazones, el tiempo de reposo de los espineles se redujo igualmente de 24 horas a aproximadamente sólo dos horas. Con relación al tipo de anzuelo utilizado, el desempeño de los diseños Mustad Kirby fue mejor a los de marca Maruto; así también, los anzuelos Mustad Kirby, presentaron menor deformación y mayor resistencia a la corrosión.

La captura total obtenida con espineles horizontales, correspondió a 77 ejemplares, con un peso total de $133,2 \mathrm{~kg}$. En la transecta frente al islote Juanango (transecta 2), las capturas fueron superiores a las generadas en el resto de las transectas $(70,4 \mathrm{~kg})$, cantidad que equivale al 52,8\% del peso total capturado. Asimismo, la transecta en la cual se determinó la menor captura en peso, fue la transecta de Bahía El Padre (transecta 3) que representa el 7,2\%. Las transectas 1 y 4, correspondientes a Puerto Francés e islote $\mathrm{El} \mathrm{Verdugo,} \mathrm{presentaron} \mathrm{respectivamen-}$ te un total de $32,4 \mathrm{~kg}$ y $20,8 \mathrm{~kg}$, equivalentes correspondientemente al $24,3 \%$ y $15,6 \%$ de la captura total (Tabla 4).

Los peces capturados con este tipo de aparejo fueron: anguila de profundidad (Bassanago nielseni Karmovskaya, 1990), breca, chancharro, corvina de Juan Fernández (Umbrina reedi Günther, 1880), morena (Gymnothorax porphyreus Guichenot, 1848), ribaldo, tollo de Juan Fernández (Squalus mitsukurii Jordan y Snyder, 1903), pez mariposa y jurel de Juan Fernández (Pseudocaranx chilensis Guichenot, 1848). Los recursos que presentaron mayor abundancia fueron el ribaldo (29), chancharro (10), breca (9), pez mariposa (9) y tollo de Juan Fernández (8).

$\mathrm{Al}$ considerar en conjunto los diversos peces que fueron extraídos por transecta con los dos aparejos utilizados en estas experiencias, se aprecia que la única especie que se encontró en todas ellas fue el ribaldo, aunque el chancharro, breca, tollo, pez mariposa y anguila de profundidad, se encontraron presentes en la mayoría de los lugares investigados. Los únicos ejemplares de corvina (1 ejemplar) y de morena (2 ejemplares), fueron capturados exclusivamente frente a Puerto Francés (transecta 1), mientras que los cuatro ejemplares de jurel de Juan Fernández, se extrajeron en la transecta ubicada perpendicularmente al islote El Verdugo (transecta 4) (Tabla 4).

Dependiendo de los diferentes estratos batimétricos en que se calaron los aparejos, la mayor variedad de especies extraídas se encontró entre los 50 y $300 \mathrm{~m}$ de profundidad. En dicho rango, las especies capturadas fueron: breca, chancharro, tollo de Juan Fernández, pez mariposa y anguila de profundidad. En 500 y 600 m sólo se extrajo el ribaldo, en tanto que los únicos ejemplares de morena $\mathrm{y}$ 
Tabla 3. Número de anzuelos calados en espineles horizontales, por estación y transecta de trabajo.

Table 3. Number of hooks set in horizontal longlines by station and working transect.

\begin{tabular}{|c|c|c|c|c|c|c|c|}
\hline & & Profundidad (m) & & ación & anz & & Total \\
\hline & & & 5 & 6 & 7 & 9 & \\
\hline & 1. Puerto Francés & 50 & 25 & 25 & 25 & 25 & 100 \\
\hline & & 100 & 25 & 25 & 25 & 25 & 100 \\
\hline & & 200 & - & - & - & - & - \\
\hline & & 300 & 15 & 15 & 15 & 15 & 60 \\
\hline & & 400 & 10 & 10 & 10 & 10 & 40 \\
\hline & & 500 & 10 & 10 & 10 & 10 & 40 \\
\hline & & 600 & 10 & 10 & 10 & 10 & 40 \\
\hline & & 800 & 10 & 10 & 10 & 10 & 40 \\
\hline & & 1000 & 10 & 10 & 10 & 10 & 40 \\
\hline & & Total & 115 & 115 & 115 & 115 & 460 \\
\hline & 2. Islote Juanango & 50 & 30 & 15 & 15 & 15 & 75 \\
\hline & & 100 & 15 & 30 & 15 & 15 & 75 \\
\hline & & 200 & 30 & 30 & 30 & 30 & 120 \\
\hline & & 300 & - & - & - & - & - \\
\hline & & 400 & 10 & 10 & 10 & 10 & 40 \\
\hline & & 500 & 10 & 10 & 10 & 10 & 40 \\
\hline & & 600 & 10 & 10 & 10 & 10 & 40 \\
\hline & & 800 & 10 & 10 & 10 & 10 & 40 \\
\hline$\stackrel{\pi}{0}$ & & 1000 & 10 & 10 & 10 & 10 & 40 \\
\hline$i_{n}$ & & Total & 125 & 125 & 110 & 110 & 470 \\
\hline 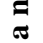 & 3. Bahía El Padre & 50 & - & - & - & - & - \\
\hline$\Leftrightarrow$ & & 100 & 10 & 10 & 10 & 10 & 40 \\
\hline & & 200 & 10 & 10 & 10 & 10 & 40 \\
\hline & & 300 & 10 & 10 & 10 & 10 & 40 \\
\hline & & 400 & 10 & 10 & 10 & 10 & 40 \\
\hline & & 500 & 10 & 10 & 10 & 10 & 40 \\
\hline & & 600 & - & - & - & - & - \\
\hline & & 800 & - & - & - & - & - \\
\hline & & 1000 & - & - & - & - & - \\
\hline & & Total & 50 & 50 & 50 & 50 & 200 \\
\hline & 4. El Verdugo & 50 & 20 & 20 & 20 & 20 & 80 \\
\hline & & 100 & 10 & 10 & 20 & 10 & 50 \\
\hline & & 200 & 5 & 5 & 5 & 5 & 20 \\
\hline & & 300 & 5 & 5 & 5 & 5 & 20 \\
\hline & & 400 & 10 & 10 & 10 & 10 & 40 \\
\hline & & 500 & 10 & 10 & 10 & 10 & 40 \\
\hline & & 600 & 10 & 10 & 10 & 10 & 40 \\
\hline & & 800 & 10 & 10 & 10 & 10 & 40 \\
\hline & & 1000 & 10 & 10 & 10 & 10 & 40 \\
\hline & & Total & 90 & 90 & 100 & 90 & 370 \\
\hline & & Total & 380 & 380 & 375 & 365 & 1500 \\
\hline
\end{tabular}


Tabla 4. Rendimiento relativo (g/anzuelo) de recursos capturados en la pesca exploratoria con espineles horizontales según la numeración del anzuelo utilizado.

Table 4. Relative yield (g/hook) by resource caught during the exploratory fishing with horizontal longlines, by station (depth) and number of the hook.

\begin{tabular}{|c|c|c|c|c|c|c|c|}
\hline \multirow[b]{2}{*}{ Transecta } & \multirow{2}{*}{$\begin{array}{c}\text { Profun- } \\
\text { didad } \\
(\mathbf{m})\end{array}$} & \multirow[b]{2}{*}{ Recurso } & \multirow{2}{*}{$\begin{array}{c}\text { Numeración } \\
\text { del anzuelo }\end{array}$} & \multirow{2}{*}{$\begin{array}{l}\text { Cantidad } \\
\text { anzuelos }\end{array}$} & \multicolumn{2}{|c|}{ Captura } & \multirow{2}{*}{$\begin{array}{c}\text { Rendimiento } \\
\text { (g/anzuelo) }\end{array}$} \\
\hline & & & & & Individuos & $\begin{array}{r}\text { Peso } \\
\text { (kg) }\end{array}$ & \\
\hline \multirow[t]{10}{*}{ 1. Puerto Francés } & \multirow[t]{2}{*}{50} & \multirow{2}{*}{$\begin{array}{l}\text { Corvina de Juan Fernández } \\
\text { Morena }\end{array}$} & 7 & 25 & 1 & 4,00 & 160,0 \\
\hline & & & 6 & 25 & 1 & 1,50 & 60,0 \\
\hline & \multirow[t]{3}{*}{100} & \multirow{3}{*}{$\begin{array}{l}\text { Breca } \\
\text { Tollo de Juan Fernández }\end{array}$} & 6 & 25 & 3 & 5,70 & 228,0 \\
\hline & & & 9 & 25 & 4 & 5,60 & 224,0 \\
\hline & & & 6 & 25 & 1 & 2,60 & 104,0 \\
\hline & \multirow[t]{2}{*}{300} & Anguila de profundidad & 9 & 15 & 5 & 6,70 & 446,7 \\
\hline & & Chancharro & 6 & 15 & 4 & 3,10 & 206,7 \\
\hline & \multirow[t]{2}{*}{600} & \multirow[t]{2}{*}{ Ribaldo } & 5 & 10 & 1 & 2,00 & 200,0 \\
\hline & & & 9 & 10 & 1 & 1,20 & 120,0 \\
\hline & \multicolumn{3}{|l|}{ Total } & 175 & 21 & 32,40 & \\
\hline \multirow[t]{26}{*}{ 2. Islote Juanango } & \multirow[t]{3}{*}{50} & \multirow[t]{3}{*}{ Pez mariposa } & 5 & 30 & 2 & 1,10 & 36,7 \\
\hline & & & 9 & 15 & 1 & 0,50 & 33,3 \\
\hline & & & 5 & 30 & 3 & 14,10 & 470,0 \\
\hline & 100 & Breca & 6 & 30 & 1 & 1,10 & 36,7 \\
\hline & & & 7 & 15 & 1 & 0,50 & 33,3 \\
\hline & & Chancharro & 6 & 30 & 2 & 1,80 & 60,0 \\
\hline & & & 9 & 15 & 1 & 0,30 & 20,0 \\
\hline & & Pez mariposa & 5 & 15 & 1 & 1,20 & 80,0 \\
\hline & 200 & Chancharro & 5 & 30 & 1 & 1,20 & 40,0 \\
\hline & & & 6 & 30 & 1 & 1,60 & 53,3 \\
\hline & & Pez mariposa & 5 & 30 & 1 & 1,00 & 33,3 \\
\hline & & & 7 & 30 & 1 & 0,90 & 30,0 \\
\hline & & & 9 & 30 & 2 & 1,15 & 38,3 \\
\hline & & Tollo de Juan Fernández & 5 & 30 & 3 & 10,40 & 346,7 \\
\hline & & & 6 & 30 & 1 & 3,20 & 106,7 \\
\hline & 400 & Anguila de profundidad & 5 & 10 & 1 & 1,60 & 160,0 \\
\hline & & Ribaldo & 7 & 10 & 1 & 1,30 & 130,0 \\
\hline & 500 & Ribaldo & 5 & 10 & 2 & 2,80 & 280,0 \\
\hline & & & 6 & 10 & 1 & 2,50 & 250,0 \\
\hline & & & 7 & 10 & 4 & 8,90 & 890,0 \\
\hline & & & 9 & 10 & 1 & 1,10 & 110,0 \\
\hline & 600 & Ribaldo & 5 & 10 & 2 & 2,80 & 280,0 \\
\hline & & & 6 & 10 & 1 & 2,50 & 250,0 \\
\hline & & & 7 & 10 & 3 & 5,70 & 570,0 \\
\hline & & & 9 & 10 & 1 & 1,10 & 110,0 \\
\hline & Total & & & 490 & 39 & $\mathbf{7 0 , 3 5}$ & \\
\hline 3. Bahía EI Padre & 200 & Pez mariposa & 6 & 10 & 1 & 0,28 & 28,0 \\
\hline & 300 & Chancharro & 7 & 10 & 1 & 0,78 & 78,0 \\
\hline & 500 & Ribaldo & 5 & 10 & 3 & 5,30 & 530,0 \\
\hline & & & 7 & 10 & 2 & 3,25 & 325,0 \\
\hline & Total & & & 40 & 7 & 9,61 & \\
\hline 4. El Verdugo & 50 & Jurel de Juan Fernández & 9 & 20 & 1 & 2,00 & 100,0 \\
\hline & 100 & Jurel de Juan Fernández & 6 & 10 & 1 & 1,80 & 180,0 \\
\hline & & & 7 & 20 & 2 & 4,60 & 230,0 \\
\hline & 500 & Ribaldo & 5 & 10 & 2 & 5,80 & 580,0 \\
\hline & & & 9 & 10 & 1 & 1,40 & 140,0 \\
\hline & 600 & Ribaldo & 7 & 10 & 2 & 4,10 & 410,0 \\
\hline & & & 9 & 10 & 1 & 1,10 & 110,0 \\
\hline & Total & & & 90 & 10 & 20,80 & \\
\hline & Total & & & 795 & 77 & 133,16 & \\
\hline
\end{tabular}


corvina de Juan Fernández fueron extraídos en el estrato de menor profundidad $(50 \mathrm{~m})$ (Tabla 4).

Al considerar globalmente la información recopilada en la pesca exploratoria con espineles horizontales, se desprende que los recursos de mayor importancia en la pesca con espineles horizontales fueron el tollo de Juan Fernández entre 50 y 200 m y el ribaldo en 500 y $600 \mathrm{~m}$. El rendimiento del tollo estuvieron comprendidos entre 104 y 470 g/anzuelo y en el caso del ribaldo entre 110 y 890 g/ anzuelo.

De los ejemplares capturados en la pesca con espinel horizontal, los rangos de longitud total y peso total, variaron de acuerdo a la especie. En el caso del tollo, las tallas estuvieron comprendidas en el rango de 75 a $91 \mathrm{~cm}$ y el peso varió entre 2,9 y 6,0 $\mathrm{kg}$. La talla mayor alcanzada por el ribaldo fue de $65 \mathrm{~cm}$ y el mayor peso de $3,2 \mathrm{~kg}$, mientras que los menores registros fueron $42 \mathrm{~cm}$ y $1,1 \mathrm{~kg}$.

Como resultado de las operaciones de pesca exploratoria con espineles en aguas circundantes a las islas Robinson Crusoe y Santa Clara, se estableció la presencia de 11 especies ícticas entre $50 \mathrm{y}$ $600 \mathrm{~m}$. Estas correspondieron a: anguila de profundidad, breca, cabrilla, chancharro, corvina, jurel de Juan Fernández, morena, ribaldo, tollo, pez mariposa y alfonsino. La mayor variedad de especies (9) fue obtenida mediante el uso de espineles horizontales, mientras que con los espineles verticales se capturaron sólo 6 especies. Sin duda este hecho estaría relacionado a las características de diseño y operación de los aparejos, así como del comportamiento de los peces antes mencionados, que evidencia que éstos se encontrarían cerca del fondo o a pocos metros sobre el piso marino.

El reducido volumen de captura obtenido globalmente puede estar asociado a las características de distribución de los recursos. Así, el calado de los aparejos obedeció a la realización de faenas de pesca en estaciones preestablecidas en un determinado lugar y profundidad, no buscando caladeros específicos para las especies encontradas. Mediante este procedimiento se pretendió conocer prioritariamente la presencia-ausencia de recursos pesqueros y la distribución geográfica y batimétrica de los mismos en torno a las islas estudiadas, que a lograr capturas significativas de cada una de ellas.

En forma tradicional los pescadores de estas islas extraen el bacalao de Juan Fernández y la breca a profundidades usualmente mayores de $50 \mathrm{~m}$ (Arana y Ziller, 1985; Arana, 1987); no obstante, en esta pesca exploratoria sólo se obtuvo este último recurso entre 50 y $200 \mathrm{~m}$. La ausencia de bacalao en las capturas sería atribuible por una parte a la paulatina disminución en la abundancia observada en este recurso y, como se mencionó anteriormente, al no dirigirse las faenas hacia los caladeros utilizados tradicionalmente por los pescadores. Por esa misma razón, es posible que no se obtuvieran otras especies que se tiene conocimiento que igualmente están presentes en estas aguas, como por ejemplo Lotella fernandeziana Rendall, 1921. Así, también, llama la atención que no se capturaran lenguados cuando se calaron los espineles en baja profundidad, lo que puede estar asociado a que se operó en fondos preferentemente rocosos, siendo más común la presencia de estos peces en fondos arenosos.

De los dos tipos de aparejos utilizados, el espinel horizontal presentó en general mayores rendimientos respecto al espinel vertical. La experiencia realizada en esta oportunidad demostró que este último tipo de aparejo puede ser empleado igualmente con éxito en estas islas. Sin embargo, desde el punto de vista operacional, el espinel vertical constituye el sistema más conveniente para ser empleado en torno a estas islas. Las características de este aparejo lo hacen especialmente apropiado para operar en aguas profundas y en fondos irregulares, ya que con ello se disminuyen las probabilidades de enredo y pérdidas de material, aunque su operación debe ser adecuada a las corrientes y al estado del mar en la zona de pesca. Esto explica que este aparejo sea ampliamente utilizado por los pescadores del archipiélago, para la obtención preferentemente de breca, tollo y bacalao de Juan Fernández.

$\mathrm{Si}$ bien las capturas obtenidas por estos aparejos no fueron especialmente abundantes, su operación permitió establecer la presencia de breca y tollo de Juan Fernández en el rango de 50 a $200 \mathrm{~m}$ y ribaldo entre 400 y $600 \mathrm{~m}$. Este último recurso es el de mayor importancia ya que hasta ahora no es pescado en estas islas, por haber presentado rendimientos de hasta 890 g/anzuelo y destacar por su carne blanca y buen sabor. Esta especie probablemente está presente en todo el cordón submarino del archipiélago de Juan Fernández, ya apareció por primera vez en la Operación Oceanográfica Pesquera MARCHILE IX en el cerro submarino O'Higgins, ubicado entre este cordón y el continente americano (Moreno, 1974; Cerda, 1977). Estos resultados destacan a este recurso como el de mayor interés para profundizar su estudio, ya que en faenas dirigidas a la pesca de esta especie es posible que se obtengan altos rendimientos. 


\section{AGRADECIMIENTOS}

El autor agradece la colaboración brindada por las tripulaciones de las chalupas de alta mar que participaron en la presente investigación. Así, también, se agradece muy especialmente al Dr. Eduardo de la Hoz U. (Q.E.P.D.), de la Universidad Católica de Valparaíso, por su aporte en la identificación taxonómica de las especies capturadas con los espineles. Finalmente, se reconoce el aporte de los evaluadores que contribuyeron en forma anónima con su experiencia a mejorar este documento.

\section{REFERENCIAS}

Arana, P. 1987. Perspectivas históricas y proyecciones de la actividad pesquera realizada en el Archipiélago de Juan Fernández, Chile. En: J.C. Castilla (ed.). Islas Oceánicas Chilenas: Conocimiento Científico y Necesidades de Investigaciones. Edic. Univ. Católica de Chile, pp. 319-353.

Arana, P. y S. Ziller. 1985. Antecedentes generales sobre la actividad pesquera realizada en el Archipiélago de Juan Fernández. En: P. Arana (ed.). Investigaciones Marinas en el Archipiélago de Juan Fernández. Esc. Ciencias del Mar, UCV, Valparaíso, pp. 125-132.

Cerda, R. 1977. Pesca exploratoria con espineles en los montes submarinos situados entre Valparaíso y archipiélago de Juan Fernández. Cienc. Téc. del Mar, CONA 3: 3-8.
Moreno, C. 1974. Mora pacífica Whaite, en el Pacífico Sur Oriental (Moridae, Gadiformes). Noticiario Mensual, Mus. Nac. Hist. Nat., Santiago, 212213: 8-10.

Pavez, P. y M.E. Oyarzún. 1985. Determinación de eficiencia relativa de espineles y parámetros de crecimiento del bacalao de Juan Fernández (Polyprion oxygeneios Bloch y Schneider, 1801), en las islas Robinson Crusoe y Santa Clara. En: P. Arana (ed.). Investigaciones Marinas en el Archipiélago de Juan Fernández. Esc. Ciencias del Mar, UCV, Valparaíso, pp. 323-340.

Pizarro, L. y E. Yáñez. 1985. Estimación de la edad de primera captura y de la mortalidad por pesca óptimas del bacalao de Juan Fernández (Polyprion oxygeneios Bloch y Schneider, 1801), a través del análisis del rendimiento por recluta. En: P. Arana (ed.). Investigaciones Marinas en el Archipiélago de Juan Fernández. Esc. Ciencias del Mar, UCV, Valparaíso, pp. 323-340.

Sepúlveda, J.I. 1987. Peces de las islas oceánicas chilenas. En: J.C. Castilla (ed.), Islas oceánicas chilenas: conocimiento científico y necesidades de investigación. Edic. Univ. Católica de Chile, pp. 235-245.

Sepúlveda, J. y G. Pequeño. 1985. Fauna íctica del archipiélago de Juan Fernández. En: P. Arana (ed.). Investigaciones Marinas en el Archipiélago de Juan Fernández. Esc. Ciencias del Mar, UCV, Valparaíso, pp. 81-91. 